\title{
QUI ÉCOUTER? QUE RECHERCHER? \\ RÉFLEXIONS D’UNE ÉTUDE DE CAS SUR LE YOGA EN INDE
}

\author{
QUEM ESCUTAR? O QUE PROCURAR? \\ REFLEXÕES A PARTIR DE UM ESTUDO DE CASO DO IOGA \\ NA ÍNDIA
}

\author{
WHO TO LISTEN TO? WHAT TO LOOK FOR? \\ REFLECTING ON A CASE STUDY ABOUT YOGA IN INDIA
}

\author{
Caroline Nizard \\ Institut d'histoire et anthropologie des religions, Faculté de théologie et de sciences des religions, Université de \\ Lausanne. Anthropole. 1015 Lausanne, Switzerland. Email: nizard.caroline@gmail.com
}

\begin{abstract}
Résumé: Malgré un travail permanent sur ses a priori culturels, sur la construction et la déconstruction des concepts et des outils méthodologiques, tout chercheur reste sous l'emprise de certaines attentes académiques et personnelles avant de partir sur le terrain. Ce récit illustre ce cheminement connu par tout scientifique qui cherche sans trouver et trouve des conclusions qu'il ne cherche pas. À travers l'exploration de terrains indiens autour de la question du yoga, je me suis interrogée sur la manière dont le milieu académique et les enquêtés européens avaient influencé mon regard et comment ces attentes s'avéraient plus admises que les réalités de terrain observées. Ainsi, les pratiquants indiens du yoga devaient se positionner dans une pratique "spirituelle" versus un bricolage psycho-corporel des pratiquants français et suisses. Or, cette mésentente colportait une définition erronée de la spiritualité, du yogi et une image galvaudée de pratiques contemporaines mondialisées.
\end{abstract}

Mots clefs: Inde, spiritualité, imaginaire, méthode ethnographique.

Resumo: Apesar de um trabalho contínuo dos investigadores sobre os seus pressupostos culturais, sobre a construção e desconstrução dos conceitos e sobre as ferramentas metodológicas a mobilizar, todos os investigadores são marcados por expectativas académicas e pessoais antes de iniciar um trabalho de investigação. Este artigo discute um processo de pesquisa no qual a investigadora procurou respostas que não encontrou, tendo encontrado outras que não procurava. Através da exploração de trabalho de terreno realizado com praticantes de ioga na Índia, Suíça e França, a autora questionou se o contexto académico e as visões reveladas pelos inquiridos europeus poderiam influenciar a visão e as expectativas da investigadora sobre as realidades observadas. Assim, esperar-se-ia que os praticantes indianos de ioga revelassem uma prática mais "espiritual” em oposição a um ajuste psico-corporal dos praticantes franceses e suíços. Este artigo revelará que estes pressupostos espelham uma definição desajustada da espiritualidade, assim como uma imagem distorcida das práticas contemporâneas mundializadas.

Palavras-chave: Índia, espiritualidade, imaginário, método etnográfico.

Abstract: Despite researchers' ongoing work on cultural preconceptions, conceptual construction and deconstruction and methodological tools, any researcher is under the influence of some academic and personal expectations before starting the fieldwork. This paper discusses the difficult process that scientists undergo while looking for answers that they do not find, and finding others which they were 
not looking for. More specifically, this paper explores fieldwork developed among yoga practitioners in India, Switzerland and France, to examine whether the reseacher's academic background and the visions revealed by the European interviewees influenced the researcher's point of view and how associated expectations could interfere in the observation of field realities. Thereby, Indian yoga practitioners were expected to reveal more "spiritual" practices while French and Swiss practitioners were expected to construct a body-psycho makeshift. However, this paper will argue that such an understanding implied a distorted perception of a depth of spirituality amongst Indian practitioners and a tarnished image of contemporary globalized practices.

Keywords: India, spirituality, fieldwork, imaginary.

\section{Qui écouter? Que voir? Cas d'un "refoulé de terrain" sur le yoga en Inde}

“C'est de l'Inde et de bien au-delà des frontières de l'Inde que viennent les dévots pour comprendre l'essence même du yoga, sa spiritualité" (Desjardins, 1961). Bien que cette citation issue du documentaire Ashram date de 1961, cet imaginaire reste particulièrement vivant tant chez les pratiquants que chez certains scientifiques.

Mon terrain parmi les pratiquants de yoga en Suisse, en France et en Inde m'a amenée à réinterroger mon positionnement sur le corps en sciences humaines. Ce terrain m'a confortée dans l'idée de dépasser les représentations sociales du corps et de franchir les limites disciplinaires en allant puiser dans d'autres sciences et méthodes. Me positionnant donc sur le chemin tantôt loué tantôt désavoué de l'interdisciplinarité, je me devais de rester dans une continuité théorique sur le yoga. De lectures en conseils avisés, j'ai ensuite exploré la question de la spiritualité et une comparaison avec l'Inde s'est (im)posée. Un état des lieux théorique approfondi a laissé apparaître une opposition entre des définitions du yoga à visée plus thérapeutique ou sportive en Europe versus une pratique plus spirituelle en Inde. Ce prédicat m'a amenée sur un premier terrain exploratoire au Karnataka (Bangalore, Mysore), Kerala (Neyyar Dam), Tamil Nadu (Pondichéry, Auroville, Chennai) pendant six mois en 2013, à la recherche du "pratiquant indien spirituel". Sur place, un nouvel objet a émergé de l'observation et des entretiens. Pour autant, il ne trouvait pas sa voix. Par la suite, un second terrain de quatre mois en 2017, à Delhi (Rajasthan) et Rishikesh (Uttarakhand), a permis de donner plus de profondeur au premier terrain indien.

Cet article souhaite discuter des influences et des possibilités d'émancipation du chercheur en sciences sociales lorsqu'il doit à la fois trouver la reconnaissance de ses pairs, donc s'appuyer sur leur savoir et à la fois être conforme au vécu des enquêtés. Cette réflexion s'articule autour d'un récit concret depuis l'élaboration d'un projet de recherche, à sa confrontation avec le terrain et dont les hypothèses 
peuvent empêcher un retour réflexif. Puis, $l^{\prime}$ article discutera des tensions entre réalités de terrain et présupposés théoriques qui interrogent le positionnement du chercheur.

\section{Retour réflexif sur la construction du terrain}

\section{Mises en perspectives théoriques ...}

“L'Inde a élaboré la technique mystique la plus systématiquement construite et là plus largement suivie que l'on connaisse: le Yoga" (Filliozat, 1946). Au yoga s'associent volontiers l'Inde et la dimension philosophique, spirituelle ou ici mystique. Dans Le yoga: Du mythe à la réalité (1991), Tardan-Masquelier déconstruit certaines représentations autour d'un yoga " $d$ 'origine", "authentique" qui n'ont plus court en Europe au vu des diverses transformations depuis sa diffusion jusqu'à aujourd'hui. De nombreux indianistes, anthropologues ont retracé les périodes de diffusion du yoga en Europe (Ceccomori, 2001; Desponds Meylan, 2007) et ses réinterprétations liées à l'influence et aux intérêts des néo-hindouistes, puis des orientalistes, de la société théosophique, des courants de la contre-culture, du New Age, et plus récemment à la sportivisation et à la consommation de masse (Mori et Squarcini, 2008). Aussi, il est communément admis que l'Europe aurait progressivement valorisé les dimensions corporelles, de santé du yoga pour le comprendre plutôt comme une pratique psycho-corporelle afin d'atteindre une sorte d'amélioration ou même de perfection teintée au gré des choix de spiritualité, de fitness, de réalisation de soi, d'ascétisme physique ou de santé (Hauser, 2013).

À cette représentation européenne et contemporaine du yoga s'oppose une représentation d'un yoga plus spirituel, philosophique avec une finalité moins corporelle en Inde. Mallinson et Singleton (2017) soulignent que le terme "yoga" désigne à la fois le but, en tant qu' union avec le divin pour se fondre en lui et se libérer du cycle des réincarnations, et le moyen, doté d'une palette de méthodes et de techniques psycho-corporelles. Ainsi, le yoga a connu une forte globalisation des pratiques où les dimensions de santé et de spiritualité restent prégnantes (Hoyez, 2012). M. Singleton (2010) a démontré des bouleversements profonds des pratiques de yoga en Inde dans les années 1920 avec une importance croissante donnée aux dimensions corporelles et sanitaires. Pour autant, dans l'imaginaire des pratiquants comme dans les milieux académiques, il semble incontournable de traiter de la spiritualité dans les pratiques de yoga en Inde. Dans la littérature, trois approches se distinguent.

L'approche sanskritiste s' intéresse à l'évolution du yoga en tant que l'une des six écoles philosophiques indiennes et pose les fondements historiques et philosophiques 
du yoga autour de textes "classiques" (Yoga Sütra, Bhagavad-Gitā, Hața Yoga Pradipikā) ou plus rares (Birch, 2011; Mallinson et Singleton, 2017). Ici, le yoga est appréhendé comme une méthode de libération du cycle des réincarnations.

L'approche historique s'illustre par de nombreuses études (anglaises ou américaines), qui se penchent de façon critique sur les périodes médiévales du yoga (White, 2012), du yoga tantrique (Samuel, 2008) ou dites de "renaissance du yoga moderne" (Alter, 2004; De Michelis, 2005; Singleton, 2010). Parmi ces derniers trava$\mathrm{ux}$, les transformations profondes notamment en termes de finalité du yoga tant en Inde que dans ses pays d'exportations (États-Unis, Europe) montrent un conflit entre une importance croissante donnée aux exercices corporels et à la dimension sanitaire et une volonté de la part des réformateurs hindous de revitaliser voire de réinventer une filiation avec la philosophie ancienne de l'Advaïta Vedanta1.

Enfin, l'approche sociologique et ethnologique s'intéresse au yoga soit en tant que "nouveau mouvement religieux" (Altglas, 2005), soit pour montrer les complexités géographiques et politiques de sa diffusion (Hoyez, 2012), soit pour se concentrer sur un courant particulier (Newcombe, 2005).

Ainsi, parmi ces trois orientations: sanskritiste, historique ou sociale, la question de la spiritualité reste au fondement des travaux sur le yoga. Les sociologues et les anthropologues s'interrogent sur les modalités de la spiritualité et de la religion dans les pratiques contemporaines, ces réflexions s'inscrivent dans le sillon creusé par les travaux de Singleton (2010), Newcombe (2017), Hauser (2013), Hoyez (2012). Pourtant, les définitions données à la spiritualité par les pratiquants européens et indiens contemporains, comme les représentations autour du yogi restent encore à explorer.

La doxa rattache la religion à une forme institutionnalisée, alors que la spiritualité donne la prévalence à l'expérience individuelle et directe, corporelle, non doctrinale. Dans le yoga, malgré des définitions qui peuvent sembler disparates, après l'analyse des discours des pratiquants suisses, français ou indiens, certaines caractéristiques émergent. Pour eux, la spiritualité (a) est une expérience individuelle, située en soi, présentée comme un "déjà-là parfois non encore ressenti". Le corps est alors réhabilité en tant que pivot de l'engagement corporel et émotionnel en impliquant autant des pratiques que des symboles. La spiritualité (b) répond majoritairement à une recherche personnelle afin de trouver des réponses à des questions existentielles avec un verbatim autour du "sens de la vie", de la "quête" ou du "chemin". Toute spiritualité dans le yoga s'inscrit dans une conception holistique du corps et de sa relation avec l'environnement (c) et renvoie à une relation personnelle avec "quelque chose" de plus grand. Ce "quelque chose" dans le langage vernaculaire peut revêtir une palette de signifiants allant de la "nature" au "grand 
Tout" comme à "Dieu" ou des "dieux", chaque émanation renvoyant toujours aux idées de non-dualisme et d'holisme. Ensuite, bien que cette spiritualité soit une transformation personnelle, elle n'est vécue que socialement (d), déjà par l'enseignant, puis par le groupe qui renforce le sentiment spirituel. Enfin, chez certains pratiquants européens rencontrés, des discours de conversion (e) peuvent apparaître. Cette définition éloigne cependant, le yoga de sa finalité sotériologique.

Suivant les travaux d'Altglas (2005) et Eisenmann (2013), je me suis tournée vers un terrain exploratoire dans les centres Sivananda en France. Dans les ashrams comme dans les cours, le rattachement à l'Inde est prégnant avec des représentations de divinités, des chants de mantra en sanskrit, des références à Mother India. Les pratiquants de ces courants construisent un rattachement sans réserve à une Inde perçue comme "spirituelle". L'ensemble de ces données issues de l'état de la recherche préalable et du terrain, a orienté le choix du terrain en Inde. Inconsciemment, je me suis mise en quête de pratiquants indiens pour qui le yoga a une visée spirituelle.

\section{...et méthodologiques avant d'aller sur le terrain}

Par mon cursus en ethnologie, $\mathrm{j}$ ' ai été nourrie par l'idée que le terrain reste au fondement de la réflexion et que la théorie aide ensuite à s'extraire de la subjectivité inhérente à l'observation participante (Beaud et Weber, 2003). Les méthodes classiques de l'anthropologie, à savoir l'observation participante et les entretiens semi-directifs, se sont donc inscrites dans une démarche inductive et dans une sociologie compréhensive (Glaser et Strauss, 1967). Si l'élaboration d'hypothèses préalables n'est pas au fondement de cette méthode, Ghasarian (2002) souligne le fait que le chercheur se forge dès le début des attentes et esquisse des questions préalables.

La grille d'entretien portait une attention particulière à trois séries d'éléments: les parcours de vie, les spécificités de l'apprentissage du yoga et les conséquences sur la vie des personnes rencontrées. Éprouvée tout au long de la recherche, elle a largement abordé les questions autour de la spiritualité, la religion, l'hindouisme, la philosophie ou les définitions du yoga. Par la suite, un va-et-vient permanent entre les terrains, les hypothèses, les analyses et les orientations théoriques a permis a posteriori de déconstruire les imaginaires et incompréhensions autour de la question de la spiritualité dans les pratiques de yogas contemporains. Ces méthodes ont permis de comprendre les significations des activités pour les pratiquants, les contradictions entre les discours et les pratiques, et ici les décalages entre les attentes parfois inconscientes du chercheur et les réalités observées et décrites. "Chaque concept, engendré ou découvert dans le processus de recherche, est dans un premier temps 
considéré comme provisoire. Il gagne sa légitimité théorique à s'avérer présent de façon répétitive [...]. Son absence peut être tout autant significative." (Strauss et Corbin, 2003, p. 366). C'est de son absence dont il sera ici en partie question.

\section{De l'élaboration du projet à la réalité du terrain}

À l'image des pratiquants de yoga contemporain qui naviguent entre les cours, mes terrains se sont concentrés sur cinq courants parmi les vingt-six recensés par Mori et Squarcini (2008). La cartographie établit par Hoyez (2012) sur les mouvements de globalisation du yoga depuis l'Inde vers l'Europe montre que certains territoires indiens se rattachent de préférence à un maître spirituel (Mysore pour Pattabhis Jois, Chennai pour Krishnamacharya, Bangalore pour Vivekananda, Rishikesh et Neyyar Dam pour Sivananda). Cette étude a orienté mes pas vers Bangalore, Mysore, Neyyar Dam, Auroville et Chennai, en Inde du Sud.

Lors de ce premier terrain exploratoire de six mois entre 2013-2014, j'ai débuté mon terrain à Bangalore. Bien que Bangalore ne soit pas réputée pour la pratique du yoga, dans l'espace public, il est aisé de trouver des centres. Je me suis rendue au centre 136.1 qui proposait des cours inspirés par de nombreux courants (satyananada, du nidra yoga ou de l'ashtanga) et qui affichait le slogan: Wellness of the mind and body awaits you. Dans ce centre, $j$ 'ai rencontré de nombreux pratiquants de yoga indiens issus plutôt de milieu aisé qui percevaient le yoga soit comme une méthode thérapeutique, soit comme une activité corporelle voire sportive, mais jamais comme une pratique philosophique ou spirituelle. Leurs discours se rapprochaient grandement de celui des Européens.

Dans le centre-ville de Bangalore, il est aussi aisé de trouver des ashrams. J'ai alors pu rencontrer des Indiens pratiquant la méditation et des chants de mantra. Je pensais toucher du doigt la spiritualité, puisqu' au centre Sivananda, ces pratiques sont désignées comme telles. Or, à ma grande surprise, ni les pratiquants, ni les responsables des ashrams ne les associaient au yoga mais à l'hindouisme en général.

Insatisfaite par ces expériences, j'ai donc suivi une formation à l'université VYASA, qui publie chaque année de nombreuses études scientifiques sur le yoga et dispense plusieurs cursus dédiés à cette discipline. Cette université s'inscrit dans un courant de yoga inspiré de Vivekananda qui est reconnu comme l'un des plus importants importateurs du yoga aux États-Unis et en Europe (Ceccomori, 2001). Vivekananda, disciple occidentalisé de Ramakrishna et l'un des fondateurs du néo-hindouisme, a simplifié et modernisé l'Advaïta Vedanta afin de le présenter comme une religion universelle. Il a allié des enseignements indiens anciens, à des éléments scientifiques occidentaux et a participé à une vision du yoga mêlant exercices corporels, philosophie et spiritualité (De Michelis, 2005). Au sein de l'Université, 
malgré une lignée se souhaitant respectueuse de sa filiation, parmi les entretiens réalisés auprès d'étudiants indiens, le lien entre yoga et spiritualité semblait flou. Ils percevaient le yoga principalement comme une méthode thérapeutique globale.

Déconcertée par ces yogas bien différents, je restais cependant dans l'expectative de rencontrer d'autres pratiquants dans le sud de l'Inde. Lors de mon séjour à Bangalore, de nombreux pratiquants indiens, sachant que j'allais me rendre plus au sud, m’ont conseillé de m'arrêter à Mysore. Mysore apparaît comme un haut lieu du yoga, notamment grâce au développement du courant ashtanga yoga dispensé à l'origine par Pattabhi Jois. L'ashtanga semble être le courant de yoga qui a connu le plus grand essor ces dernières années en Europe et aux États-Unis. Il se caractérise par son dynamisme, sa dimension beaucoup plus physique, voire sportive et sa structure en séries qui suivent des enchaînements strictement identiques. La popularité internationale de ce courant témoigne des mouvements de globalisation autour des pratiques de yoga, car Pattabhi Jois est mieux connu à l'étranger qu'en Inde. La majorité des étrangers qui se rendent à Mysore sont cadres supérieurs, ingénieurs ou en reconversion pour devenir professeurs de yoga. À Mysore, le yoga est une industrie florissante. Les Indiens pratiquant le yoga, majoritairement professeurs ou en devenir, souhaitent à la fois dispenser un enseignement dans la lignée de Pattabhis Jois et profiter des retombées économiques de ce marché. De nombreux centres de yoga se sont formés aux alentours du centre de Jois. Comme les cours se terminent tôt, le reste de la journée peut être consacré à approfondir d'autres dimensions du yoga avec des cours de philosophie hindoue, de chants de mantras ${ }^{2}$, notamment.

Après avoir rencontré majoritairement des étrangers à Mysore, je me suis aussi rendue dans un ashram pendant une semaine dans un centre Sivananda à Neyyar Dam. Ayant déjà participé à une retraite dans un ashram Sivananda en France à côté d'Orléans, les similarités étaient frappantes et démontrent la volonté de ce courant d'homogénéiser et d'exporter à l'identique le contenu des enseignements. Tout d'abord, le centre se situe au milieu de la nature, $l^{\prime}$ emploi du temps est exactement le même, la structure des cours analogues. Le centre reste peu fréquenté par les Indiens, à l'exception de quelques professeurs et membres du personnel. Les discours des pratiquants à Neyyar Dam étaient en de nombreux points similaires à ceux d'Orléans et je me suis interrogée sur la nécessité de parcourir autant de kilomètres pour recueillir les mêmes témoignages.

Mes autres terrains indiens n'ont pas fait apparaître d'autres pratiques malgré la diversité des courants observés et des entretiens réalisés. À Auroville, Mysore ou Neyyar Dam, j'ai interrogé plus de pratiquants étrangers qu'Indiens. Aussi, au fil des kilomètres, j' ai constaté que les centres portant un discours affiché comme "spirituel" étaient majoritairement fréquentés par des Européens ou des Américains. En 
revanche, à la lumière des quinze entretiens réalisés auprès de pratiquants indiens du yoga, tous témoignaient de définitions du yoga éloignées de la spiritualité, mais dont la finalité était d'abord sanitaire et corporelle. Ainsi, les Indiens rencontrés avaient des discours en de nombreux points similaires aux pratiquants rencontrés en France et en Suisse romande.

Ces similitudes s'expliquent en partie par des caractéristiques socio-culturelles communes. En effet, les pratiquants rencontrés en France et en Suisse romande sont pour les trois quarts des femmes ayant fait des études supérieures et occupant des postes à responsabilités élevées, avec une majorité de personnes travaillant dans les milieux médicaux, sociaux, des cadres ou des professions libérales. Leur moyenne d'âge se situe autour de 40 ans. Cependant, les Indiens rencontrés étaient des mêmes milieux sociaux, mais dans l'ensemble plus âgés. Les cours étaient fréquentés par plus d'un tiers d'hommes. Les caractéristiques socio-professionnelles expliquent en partie les similitudes entre les motivations de ces urbains stressés en quête de détente. Pourtant, elles n'expliquent pas la place différente prise par la spiritualité dans les pratiques du yoga sachant que les enseignements au sein d'un même courant demeurent très proches.

Dans le discours des pratiquants indiens, les pratiques étiquetées par les Européens comme "spirituelles" commel'enseignement philosophique, les chants de mantra, la méditation, étaient toujours perçues comme des éléments appartenant à la culture indienne et non propres au yoga. Pour les Indiens rencontrés, le yoga ne participe pas à un "éveil spirituel", à une quête de sens. Aussi, le yoga n'apportait sur ces plans aucun changement dans leurs comportements. Par ailleurs, j'ai été frappée par l'apprentissage de techniques du corps quasiment identiques au sein de chaque courant de yoga. En effet, un cours d'ashtanga se déroule selon le même schéma à Genève, Paris ou Mysore. Il existe plus de différences entre les courants qu'entre les pratiquants indiens et européens. Aussi, malgré une collecte de données importantes, un carnet de terrain, à mon retour, $\mathrm{j}^{\prime}$ ai considéré ce terrain comme "raté", supposant que je n' avais pas réussi à rencontrer les bons interlocuteurs. À mon retour, j' ai exclu l'Inde et ce terrain de mes recherches en me recentrant sur la France et la Suisse.

\section{Construction inconsciente d'un a priori scientifique}

\section{Confrontation entre élaboration théorique et observation concrète}

Alors que je me consacrais à d'autres aspects de ma recherche, j'ai aussi participé à de nombreuses conférences sur le yoga. C'est alors que j'ai pris conscience de l'a 
priori partagé par certains scientifiques, souvent non spécialistes du yoga, et la majorité des pratiquants européens, à savoir l'idée que les pratiquants de yoga indiens inscrivent d'emblée leur pratique dans une démarche spirituelle.

Alors que je commençais à écrire, les discours des pratiquants indiens sont venus hanter mes écrits, m'empêchant de me concentrer uniquement sur la France et la Suisse. L'introduction de V. Bouillier et G. Tarabout (2002) a mis au jour ce paradoxe resté latent d'un orient spirituel versus un occident matérialiste. Elle est alors entrée en résonance avec un entretien d'un professeur indien rencontré à Chennai. Ce dernier soulevait une opposition entre les Indiens qui étaient de plus en plus attirés par la société de consommation et la matérialité, fascinés par l'ouest, tandis que les occidentaux venaient toujours plus nombreux en Inde à la recherche de la spiritualité.

C'est grâce à ce double écho, que je me suis rendue compte de mes a priori qui étaient devenus mes prédicats de recherche. $S^{\prime}$ ils semblent clairs aujourd'hui, ils ne l'étaient pas lors du premier terrain. Alors que, en "bons" ethnologues nous veillons à travailler sur nos inconscients culturels, j'avais occulté la persistance de l'imaginaire exotique chez les pratiquants européens ainsi que les présupposés intellectuels des milieux scientifiques.

S'il s' efforce d'entrer sur le terrain avec un "esprit ouvert", l' ethnographe, quelles que soient ses bonnes intentions, n'est pas exempt de préconceptions et de présupposés (que l'on trouve déjà dans le choix du sujet, du lieu géographique et des personnes à étudier). Non contrôlées, c'est-à-dire non explicitées, ces préoccupations peuvent saper la recherche (Ghasarian, 2002, pp. 10-11)

Je m'étais ainsi construit l'image du pratiquant indien que je devais rencontrer, et tout cela avait voyagé dans mes bagages, au point d'en construire un "refoulé de terrain". Me détachant alors provisoirement de mes positionnements théoriques sur le yoga, $\mathrm{j}$ ' ai fait confiance à mes intuitions et autorisé un "retour du refoulé" de terrain. L'intérêt du terrain indien est apparu alors non pas dans sa confirmation des dimensions spirituelles du yoga sur place, mais au contraire dans ses visées pragmatiques très similaires à celles de l'Europe.

Là réside aussi une ambiguïté propre à l' anthropologie contemporaine qui a progressivement autorisé le chercheur à s'intéresser à des terrains proches et non plus lointains. Cependant, dès que l'anthropologue s'engage sur un terrain culturellement lointain, il reste l'idée de devoir s'y confronter, $\mathrm{d}^{\prime}$ "aller expérimenter la différence" (Caratini et Godelier, 2012, p. 16), de chercher les particularités (Abélès dans Ghasarian, 2002). Alors que l'Inde présente aujourd'hui de nombreux objets de recherches pour l'ethnologue souhaitant s'intéresser à des 
spécificités culturelles et des différences, face aux pratiquants de yoga rencontrés, il y avait, du fait de la diffusion de la pratique, de la globalisation de ces techniques du corps, mais aussi du va-et-vient entre l'Inde et l'Occident, de nombreuses similitudes. Appadurai (2001) a déjà déconstruit de nombreux $a$ priori sur l'Inde alors que les modes de circulation liés aux interactions mondiales contemporaines, les différences géographiques et culturelles tendent à s'effacer. Bien que tout chercheur soit aujourd'hui conscient des transformations sociales de toutes les sociétés, de l'impact de la globalisation, de l'influence $\mathrm{d}$ 'internet, des outils de communication, il reste encore difficile de concevoir cet ailleurs comme plus proche qu'étranger (Ghasarian, 2002).

\section{Retour sur le terrain en Inde du Nord}

Grâce à ces nouvelles perspectives et laissant de côté cette recherche du pratiquant d'un yoga spirituel en Inde, j' ai entrepris un deuxième terrain en 2017 qui a confirmé ces premières observations. Il a cependant permis de souligner à la fois des points communs et des différences non dans les pratiques, mais dans les parcours de vie et dans le sens donné à la pratique. Contrairement à mon premier terrain, où je n'avais aucun informateur préalable, grâce à mon réseau, j'ai été mise en contact avec un professeur de yoga à Delhi. Cela a déterminé le choix des lieux d'observation et a permis une immersion de quatre mois en 2017 au sein du Yoga Studio. Ce professeur ne se rattache pas à un courant spécifique et mélange les styles Iyengar, Sivananda et Ashtanga.

Au sein du centre, mon double statut de chercheure et de pratiquante de yoga était connu de tous, la responsable m'ayant présentée pour faciliter la tenue des entretiens. J'ai pu ainsi recueillir une quinzaine d'entretiens. Les caractéristiques d'âge, de genre ou de milieux socio-professionnels étaient identiques à celles des personnes rencontrées en Inde du Sud auparavant.

Enfin, poussée par la lecture de nombreux travaux sur Rishikesh qui est considérée aussi bien par l'État indien, les guides de voyages ou les pratiquants du yoga comme l'un des lieux incontournables pour aller faire du yoga en Inde, j' ai décidé de participer à un festival de yoga organisé chaque année par l'Ashram Parmarth Niketan. Ironie du sort ou pied de nez du terrain, à Rishikesh, alors que ce deuxième terrain s'achevait, j'ai aussi rencontré des pratiquants indiens pour qui la dimension spirituelle dans le yoga était importante. La boucle était bouclée mais avec des conclusions assez différentes. Ce dernier terrain a donné la pièce manquante du puzzle et éclairé deux confusions importantes autour de la spiritualité et l'image du pratiquant. 


\section{Observations et remarques}

Les entretiens à Delhi ont confirmé les conclusions du premier terrain indien. En résumé, pour ces pratiquants rencontrés, le yoga apparaît en premier lieu comme une méthode thérapeutique. La santé apparaît une motivation beaucoup plus forte chez les Indiens que chez les Européens rencontrés, car les médecins orientent leurs patients vers le yoga. Comme pour de nombreux européens, la détente, le bien-être jouent un rôle important, sans être une motivation première. Le yoga s'apparente à une manière de prendre soin de soi, de se maintenir en bonne santé.

Il existe de fortes résonnances entre les discours des Français, des Suisses et des Indiens rencontrés. Ainsi, il a été possible d'observer plusieurs itinéraires de pratiques. Certains ont une pratique de loisir et utilisent le yoga comme une boîte à outils permettant de régler des désagréments du quotidien (stress, mal de dos, etc.). D'autres s'engagent dans un processus de transformations plus profond. Le yoga prend alors une place importante dans leur vie et apporte de nombreux changements (corporels, alimentaires, dans la gestion des émotions, etc.). Les parcours se singularisent selon les choix et les motivations premières. Enfin, les passionnés voient leur quotidien totalement transformé. Il se met en place une véritable hygiène de vie, leur vie tourne autour du yoga et toutes les sphères de leur vie sont impactées. Pour ces derniers, le yoga apparaît à plus d'un titre comme une orthopraxie.

Si je m'étais arrêtée à l'observation participante sans avoir procédé à des entretiens approfondis, $\mathrm{j}$ 'aurais pu conclure qu'il existe en Inde comme en Europe des courants plus portés sur la dimension spirituelle que d'autres. Pourtant, les entretiens ont permis de mettre au jour des différences dans le sens donné aux pratiques. Les incompréhensions concernant la question de la spiritualité relèvent (1) de la qualification de certaines pratiques, (2) de leur rattachement au yoga et (3) de la distinction entre yogi et pratiquant de yoga.

Alors qu'avec les Français et les Suisses rencontrés, la spiritualité apparait souvent dans les discours, si je n'abordais pas la question directement avec les Indiens, aucune personne interrogée ne faisait spontanément un lien entre yoga et spiritualité. Parmi les Indiens interrogés, certains se définissent comme hindous, musulmans, sikhs et déclarent pratiquer des rituels, aller aux temples, notamment. Généralement, ces personnes croient, ressentent une spiritualité et ont une religion. Pour autant, leur définition de la spiritualité se distingue sur deux points: la spiritualité répond rarement pour eux à une "quête de sens". Par ailleurs, aucun indien rencontré n'a évoqué une "conversion", ce dont témoigne certains pratiquants européens. 
Pour les Indiens, la méditation comme le chant de mantras sont déjà présents dans d'autres pratiques rituelles. Les nombreuses divinités du panthéon indien ne se rattachent pas spécifiquement au yoga. Au mieux, le yoga est une expression de leur héritage culturel, mais n'ajoute rien à la spiritualité ressentie. La spiritualité s'exprime dans les rituels, le fait d'aller dans les temples, d'effectuer des offrandes, bien plus que dans la pratique du yoga.

Ces distinctions dans le sens donné aux pratiques peuvent paraître subtiles. Pourtant, elles expliquent pourquoi en tant qu' européenne je percevais des signes, des pratiques que j'aurais qualifiées de "spirituelles", "religieuses", alors qu'aux yeux des Indiens elles n'apparaissent pas comme telles, dans le cadre du cours de yoga. Par ailleurs, la manière de poser l'hypothèse était erronée. Il ne s'agissait pas de trouver des pratiquants de yoga indiens avec des pratiques plus "spirituelles" en Inde versus des pratiques corporelles en Europe, car les spécificités culturelles ne résident pas dans les actions, mais dans le sens donné à ces actes. Ainsi, par exemple, s'il est possible d'observer des pratiquants de yoga indiens chanter des mantras, pour eux, cet acte n'apparaît pas comme une spiritualité associée au yoga, alors que pour un européen, chanter des mantras relève d'une activité extraordinaire découverte dans les cours de yoga et s'associant aux dimensions spirituelles de cette pratique.

Grâce à l'approfondissement des recherches, j'ai compris un troisième malentendu: la non-distinction entre pratiquant de yoga et yogi. Autant le terme de "yoga" a fait l'objet de nombreuses critiques, autant celui de "yogi" qui désigne aujourd'hui une personne qui pratique le yoga reste peu discuté. En effet, les yogis se réfèrent à des ascètes hindous, des renonçants au monde, dont certains ont développé les premières postures de yoga. Parmi ces renonçants (sannyasin ou sadhus), certains ordres existent toujours en Inde. Les Nāth, étudiés par Véronique Bouillier (2008), ne pratiquent que rarement les postures (ou seulement certaines), mais utilisent bien le yoga comme méthode sotériologique. D'autres renonçant restent dans des postures pendant plusieurs mois ou années, par exemple en gardant une main en l'air ou en restant sur une jambe (Mori et Squarcini, 2008). Enfin, d'autres communautés d'ascètes (Vaisnava, Nāth ou Tantrika) nomment yoga, le chemin et l'effort pour atteindre un but (Bevilacqua, 2017; Mallinson, 2016). Ces pratiques qualifiées de tapas, ont pour finalité d'obtenir un discernement, une avancée spirituelle ou des pouvoirs surnaturels. Ainsi, le yoga moderne se traduit par un éventail de pratiquants allant des ascètes vivant seuls ou en communautés fermées, à des gourous connus pour leur sens des affaires (Baba Ramdev ou Yogi Bhajan), en passant par des courants plus ou moins institutionnalisés et des cours de yoga autour d'un professeur faisant des mélanges personnels et hybrides (Newcombe, 2017). 
Ainsi autour de ce yogi s'est créée un yogi imaginaire, sorte d'idéal à atteindre (d'élévation spirituelle et d'avancée dans la méditation), mais qui se confond avec la majorité des pratiquants de yoga. Le pratiquant de yoga étant celui qui utilise un ensemble de techniques psycho-corporelles de postures, de respiration et/ou de méditation et qui ne cherche pas forcément une délivrance, mais une harmonie entre corps et esprit. Les pratiquants passionnés cherchent à atteindre ce perfectionnement de soi en s'imposant une orthopraxie, cependant ils ne donnent ni au yoga, ni à la spiritualité la même finalité. En s'accordant sur ces définitions, les Indiens, Français et Suisses rencontrés devraient tous être qualifiés de pratiquants de yoga, même lorsqu'ils s'autodéfinissent comme des "yogis".

Au départ, dans mon imaginaire, la superposition de ces deux figures n'était pas claire, d'où un autre niveau d'incompréhension sur ces représentations de la spiritualité et du yoga contemporain en Inde. Nonobstant, exclure les pratiquants indiens qui utilisent des pratiques et des représentations du yoga globalisées et uniformisées seraient occulter une réalité très vivante, voire majoritaire en Inde aujourd'hui.

\section{Conclusion}

Aujourd'hui, lorsque je présente ces conclusions auprès de spécialistes sur l'Inde, je me retrouve confrontée à cet imaginaire. J'avance alors des extraits d'entretiens, limite ces conclusions aux personnes rencontrées. Comme le yoga est une discipline connue de tous, même les non-spécialistes du yoga dans la recherche ont une opinion sur cette question sans forcément s'interroger sur leurs propres représentations. Ainsi, ils laissent de côté leurs lunettes de scientifiques pour se construire une image du yoga.

Jeanne Favret-Saada et Josée Contretas (1981) ou Louis Dumont (1951) ont déjà mis en garde contre certaines idées reçues sur l'humain, la sorcellerie ou d'autres notions, non seulement au sein de la population étudiée, mais aussi dans la communauté scientifique. En effet, s' intéresser à certains sujets induit obligatoirement une orientation préétablie par les recherches antérieures. Alors que tout anthropologue valorise la possibilité que le terrain remette tout en cause, dans les faits, il reste compliqué de dépeindre une réalité non attendue, en décalage avec les attentes, surtout pour le jeune chercheur. Méthodologiquement, il conviendrait d'analyser les comportements, mais aussi les représentations des enquêtés comme des scientifiques. Chacun porte un regard, une traduction (Ghasarian, 2002), les premiers de leurs ressentis et de leurs imaginaires autour du yoga, les seconds autour de leurs recherches, leurs hypothèses et conclusions qui forment une traduction de la réalité. 
Le récit de la construction, de la définition des hypothèses et de la restitution de ces quelques conclusions permet non pas de s'attarder sur le cas précis du yoga, mais de montrer l'influence de l'imaginaire des enquêtés, des présupposés théoriques et académiques qui existent dans toute recherche. Alors que l'anthropologue veille à déconstruire ses présupposés culturels, à laisser au terrain la chance de s'exprimer, parfois il n'a pas la capacité de l'entendre. Ici, la recherche théorique préalable, l'influence de travaux pertinents ont orienté mes interprétations, empêchant dans un premier temps, les données empiriques d'émerger. Par la suite, l'originalité s'est révélée plus dans les similitudes que dans les différences dans les pratiques de yoga en France, en Suisse et en Inde. Ainsi, en comprenant ces pratiques comme globalisées, ces techniques du corps comme de plus en plus uniformisées au sein d'un courant, il a été possible de retourner sur le terrain. Ce va-et-vient a permis de confirmer ces points communs tout en apportant des nuances dans le sens donné aux pratiques. La persévérance permet de mettre au jour des constructions culturelles intéressantes et de donner un sens à des terrains en décalage avec les attentes.

\section{Notes}

1 Il s'agit d'un courant fondé à l'époque médiévale par Shankara, valorisant la non-dualité, c' est-à-dire que l'Absolu divin (Brahman) serait unifié aux âmes individuelles (ātman). Ici, la libération du cycle des réincarnations revient à dépasser toute opposition conflictuelle toute dualité, pour accéder au seul infini. Ce courant s'est ensuite imposé comme une version officielle de l'hindouisme dans la bourgeoisie indienne de l'époque coloniale, car il lui donnait une vision unitaire et respectable.

2 À Mysore, comme à Chennai au centre Krishnamacharya, où je me suis rendue par la suite, les responsables des centres ne veulent pas enseigner la philosophie ou dispenser des enseignements "spirituels". Ils estiment qu'il s'agit d'un cheminement personnel et que chacun peut, à travers la transformation occasionnée par le yoga, découvrir.

\section{Références}

Alter, J. (2004). Yoga in modern India, the body between science and philosophy. Princeton et Oxford: Princeton University Press.

Altglas, V. (2005). Le nouvel hindouisme occidental. Paris: CNRS Éditions.

Appadurai, A. (2001 [1996]). Après le colonialisme. Les conséquences culturelles de la globalisation. Paris: Payot.

Beaud, S., et Weber, F. (2003). Le guide de l'enquête de terrain: Produire et analyser des données ethnographiques. Paris: Éditions La Découverte. 
Bevilacqua, D. (2017). Let the Sadhus talk: Ascetic understanding of hatha Yoga and yogasanas. Religious of South Asia, 11(2-3), 182-206.

Birch, J. (2011). The meaning of hatha in early hathayoga. Journal of the American Oriental Society, 131(4), 527-554.

Bouillier, V. (2008). Itinérance et vie monastique: Les ascètes Nāth Yogis en Inde contemporaine. Paris: Editions de la Maison des Sciences de l'Homme.

Bouillier, V., et Tarabout, G. (Dir.) (2002). Images du corps dans le monde hindou. Paris: CNRS éditions.

Caratini, S., et Godelier, M. (2012). Les non-dits de l'anthropologie. Vincennes: Éditions Thierry Marchaisse.

Ceccomori, S. (2001). Cent ans de yoga en France. Paris: Édidit.

De Michelis, É. (2005). A history of modern yoga, Patañjali and Western esotericism. Londres, New York: Bloomsbury Academic.

Desjardins, A. (2006 [1961]). Ashram. Les Ollières-sur-Eyrieux: Alizé diffusion.

Desponds Meylan, S. (2007). L'enseignement de yoga européen entre adhikara et pédagogie. Une analyse de la qualification socio-religieuse des enseignants dans la rencontre entre l'Union européenne de yoga et le lignage de T. Krishnamacharya (Thèse). Faculté des lettres de l'université de Lausanne, Suisse.

Dumont, L. (1951). La tarasque. Essai de description d'un fait local d'un point de vue ethnographique. Paris: Éditions Gallimard.

Eisenmann, C. (2013). Embodied spirituality in Yoga practice. Conference paper.

Favret-Saada, J., et Contretas, J. (1981). Corps pour corps. Enquête sur la sorcellerie dans le bocage. Paris: Éditions Gallimard.

Filliozat, J. (1946). Les origines d'une technique mystique indienne. Revue philosophique de la France et de l'étranger, 136(4/6), 208-220.

Ghasarian, C. (2002). De l'ethnographie à l'anthropologie réflexive. Nouveaux terrains, nouvelles pratiques, nouveaux enjeux. Paris: Armand Colin.

Glaser, B., et Strauss, A. (1967). The discovery of grounded theory: Strategies for qualitative research. Chicago: Aldine Publications.

Hauser, B. (Ed.). (2013). Yoga traveling. Bodily practice in transcultural perspective. New York: Cham, Springer.

Hoyez, A.-C. (2012). L'espace-monde du yoga. De la santé aux paysages thérapeutiques mondialisés. Rennes: Presses Universitaires de Rennes.

Mallinson, J. (2016). The Hața Yoga project. Disponible à http://hyp.soas.ac.uk/ .

Mallinson, J., et Singleton, M. (2017). Roots of Yoga. Londres: Penguin Books.

Mori, L., et Squarcini, F. (2008). Fra yoga, storia e mercato. Rome: Carocci.

Newcombe, S. (2005). Spirituality and 'mystical religion' in contemporary society: A case study of British practitioners of the Iyengar method of yoga. Journal of Contemporary Religion, 20(3), 305-322.

Newcombe, S. (2017). The revival of Yoga in contemporary India. Dans Oxford Research Encyclopedia of Religion. USA: Oxford University Press. 
Samuel, G. (2008). The origins of Yoga and Tantra, Indic religions to the thirteenth century. Cambridge: Cambridge University Press.

Strauss, A., et Corbin, J. (2003). L'analyse de données selon la 'grounded theory'.

Procédures de codage et critères d'évaluation. Dans D. Céfaï (Ed.), L'enquête de terrain (pp. 363-379). Paris: Les Éditions la découverte.

Singleton, M. (2010). Yoga body. The origins of modern posture practice. Oxford: Oxford University Press.

Tardan-Masquelier, Y. (1991). Le yoga: Du mythe à la réalité. Paris: Droguet et Ardent.

White, D. G. (2012). Yoga a brief history of an idea. Princeton: Princeton University Press.

Data de submissão: 23/04/2019 | Data de aceitação: 01/07/2019 